\title{
Clinical Heterogeneity in Acute Symptomatic Seizures due to Autoimmune Encephalitis Related to GAD65 Antibodies
}

\author{
Xuemei Fan ${ }^{a}$ Xinxin $\mathrm{Li}^{\mathrm{a}}$ Shuai Wang ${ }^{\mathrm{b}}$ Yafei Zhu ${ }^{\mathrm{a}}$ Songyan $\mathrm{Liu}^{\mathrm{a}}$ \\ Xuemei Han ${ }^{\mathrm{a}}$ \\ aDepartment of Neurology, China - Japan Union Hospital of Jilin University, Changchun, China; ${ }^{\mathrm{b}}$ Department of \\ Gastroenterology, China - Japan Union Hospital of Jilin University, Changchun, China
}

\section{Keywords}

Glutamate decarboxylase 65 antibody · Autoimmune encephalitis · Acute symptomatic seizures

\section{Abstract \\ Introduction: This study aimed to explore the diversity and clinical features of acute symptomatic seizures due to auto- immune encephalitis related to anti-glutamate decarboxyl- ase (GAD) 65 antibodies. Methods: Clinical data of a series of 6 patients positive for anti-GAD65 antibodies were retro- spectively analyzed. Results: Five of the patients were male and 1 was a female, with a median age of 44.1 years (range 18-70 years). Seizure forms were varied in 6 patients when they were admitted to the hospital: 3 cases of seizures only and 3 accompanied by other symptoms, such as mental dis- order, cognitive impairment, cerebellar ataxia, and ocular movement disorder. Three patients (50\%) had coexisting systemic autoimmune diseases, including diabetes mellitus, vitiligo, and hyperthyroidism. Five patients (83\%) had abnor- mal brain MRI findings. They were all treated by immuno- therapy, 5 of 6 patients improved significantly but relapsed after withdrawing methylprednisolone, and 1 patient got}

deteriorated. None of them were diagnosed with tumors. Conclusions: Clinical features of acute symptomatic seizures related to GAD65 antibodies are diverse, and early and continuous immunotherapy is necessary for patients.

(c) 2021 The Author(s).

Published by S. Karger AG, Basel

\section{Introduction}

Acute symptomatic seizures are one of the most common symptoms of GAD65 AE. The most recent International League Against Epilepsy (ILAE) supports using the term "acute symptomatic seizures secondary to AE" to refer to seizures occurring in the setting of the active phase of immune-mediated encephalitis and suggests the term "autoimmune-associated epilepsy" to refer to chronic seizures determined to be secondary to autoimmune brain diseases [1]. In this study, the clinical symptoms, auxiliary examinations, treatments, and prognosis of 6 patients with GAD65 antibody-associated acute symptomatic seizure were discussed and analyzed to improve the understanding of the disease.
C 2021 The Author(s).

Published by S. Karger AG, Basel

This is an Open Access article licensed under the Creative Commons Attribution-NonCommercial-4.0 International License (CC BY-NC) (http://www.karger.com/Services/OpenAccessLicense), applicable to the online version of the article only. Usage and distribution for commercial purposes requires written permission.
Correspondence to:

Songyan Liu, liu_sy@jlu.edu.cn

Xuemei Han, hxm@jlu.edu.cn 
Table 1. Data of 6 patients positive for GAD65 antibody

\begin{tabular}{|c|c|c|c|c|c|c|}
\hline Patient & 1 & 2 & 3 & 4 & 5 & 6 \\
\hline Age, sex & $61, M$ & $44, F$ & $18, M$ & $23, \mathrm{M}$ & $49, M$ & $70, M$ \\
\hline Disease form & $\begin{array}{l}\text { Partial sensory and } \\
\text { autonomic seizures }\end{array}$ & GTCS & NORSE & $\begin{array}{l}\text { Partial sensory, } \\
\text { particle motion }\end{array}$ & FBTCS, SE & $\begin{array}{l}\text { Particle } \\
\text { motion, SE }\end{array}$ \\
\hline Clinical features & $\begin{array}{l}\text { Depression, memory } \\
\text { impairment }\end{array}$ & None & Diplopia, CA & Dysarthria & $\begin{array}{l}\text { Conscious } \\
\text { disturbance }\end{array}$ & None \\
\hline $\begin{array}{l}\text { Disease duration } \\
\text { GAD Ab titers }\end{array}$ & 8 months & 3 years & 7 days & 14 days & 7 days & 12 days \\
\hline Serum & $1: 100$ & $1: 320$ & $1: 32$ & $1: 100$ & $1: 32$ & $1: 100$ \\
\hline CSF & $1: 100$ & $1: 100$ & $1: 1$ & $1: 32$ & $1: 100$ & $1: 10$ \\
\hline Other autoantibodies & $\begin{array}{l}\text { LGI1, ICA: (+) } \\
\text { AMA-M2: (+) } \\
\text { ANA: } 1: 100\end{array}$ & $\begin{array}{l}\text { ICA: (+), A-TG: }(+), \\
\text { A-TPO: }(+)\end{array}$ & $\begin{array}{l}\text { A-TG: }(+), \text { A-TPO: }(+), \\
\text { CCP }(+), \text { ANA: } 1: 100\end{array}$ & - & - & - \\
\hline \multicolumn{7}{|l|}{ CSF } \\
\hline Grave's & - & + & + & - & - & - \\
\hline Vitiligo & + & - & - & - & - & \\
\hline Neoplasm & - & - & - & - & - & - \\
\hline Immunotherapy & IVMP + IVIG & IVMP + IVIG & IVMP + IVIG & IVMP + IVIG & IVMP & IVMP \\
\hline Pre-QOLIE-31 & 58 & 55 & - & - & - & 60 \\
\hline Post-QOLIE-31 & 89 & 86 & 67 & 72 & 0 & 86 \\
\hline
\end{tabular}

F, female; M, male; GTCS, generalized tonic-clonic seizures; SE, status epilepticus; NORSE, new-onset refractory status epilepticus; FBTCS, focal to bilateral tonic-clonic seizures; IVMP, intravenous methylprednisolone; IVIG, intravenous immunoglobulin; WBC, white blood cell; CSF, cerebrospinal fluid; QOLIE-31, 31-item quality of life in epilepsy.

\section{Materials and Method and Patients}

From May 2018 to December 2020, 6 patients, including one with coexistence of anti-leucine-rich glioma-inactivated protein (LGI1) antibody, who were positive for anti-GAD65 antibodies were diagnosed and admitted to the Department of Neurology, the Hospital of Jilin University, Changchun, China. The study was approved by the Ethics Committee of China - Japan Union Hospital of Jilin University. All patients were informed and provided informed consent to participate in the study.

\section{Method}

Complete clinical data were collected after admission including the results of neurological, serum laboratory, cerebrospinal fluid, neuroelectrophysiology, and MRI examinations. Serum and cerebrospinal fluid samples were obtained from all patients for the detection of autoimmune encephalitis (NMDA, LGI1, AMPA1, AMPA2, GABA, CASPR2, and GAD65) and paraneoplastic (Ma2, Hu, Yo, Ri, CV2, CRMP5, and amphiphysin) antibodies. All samples were measured using Indirect Immunofluorescence Test (IIFT) kits purchased from EUROIMMUN AG (Lübeck, Germany) and used according to the manufacturer's instructions.

\section{Curative Effect Evaluation}

We used the 31-item quality of life in epilepsy (QOLIE) inventory - QOLIE-31 - to assess epilepsy persons's overall wellness including seizure worries, cognitive impact, and social functioning, and details of the scoring system are provided in the QOLIE-31 Scoring Manual [2]. A composite score from 0 to 100 that represents higher scores represent better function.

\section{Results}

The clinical data of 6 patients are summarized in Table 1 . Five of the patients were males and 1 was a female, with a median age of 44.1 years (range 18-70 years).

Seizure forms were varied in 6 patients; 3 of 6 patients developed fever $\left(37.1-38.2^{\circ} \mathrm{C}\right)$ before seizure, and in addition to seizures, they demonstrated other symptoms, such as mental disorder, cognitive impairment, CA, and ocular movement disorder. One patient developed rapidly progressive memory and affective disturbances 4 months after the seizure, while the other 2 pa- 

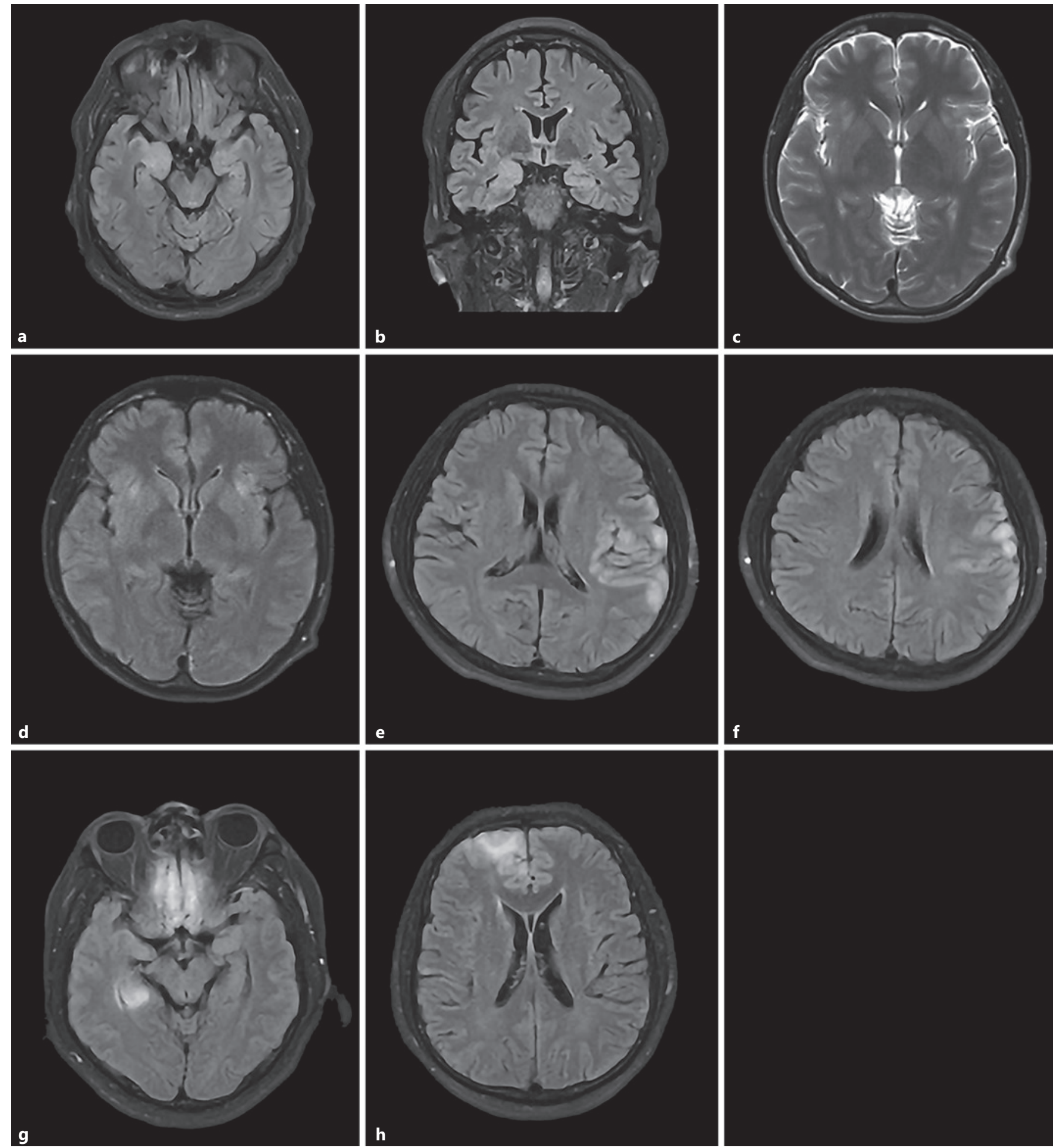

Fig. 1. a (Pt 1) The right amygdala hyperintensity and enlargement in MRI FLAIR weighted; the left hippocampal sclerosis and atrophy (b). c, d (Pt 3) Hyperintense in the bilateral insula cortex in MRI T2 and FLAIR weighted. e, $\mathbf{f}(\mathrm{Pt} 4)$ Hyperintense in the left parietal, temporal, and the insula cortex. $\mathbf{g}, \mathbf{h}$ (Pt 5) Hyperintense in the right temporal, bilateral frontal lobes, and the frontopolar cortex. 
tients presented only seizures all the time. Patient 1 had $\mathrm{DM}$ and vitiligo, and patient 2 had DM and hyperthyroidism.

We noted coexistence of anti-LGI1 antibodies and anti-GAD65 antibodies in patient 1 . Patient 3 had serological evidence of multiple autoantibodies (anti-TPO, antinuclear, and anti-cyclocitrulline antibodies), but without any clinical signs of autoimmune diseases. No paraneoplastic antibody was detected in all patients. Brain MRI usually showed hyperintensity and "swelling" in medial temporal structures in T2/FLAIR MRI sequences, hippocampal sclerosis, and atrophy (Fig. 1a-d) and hyperintense cortical/subcortical lesions predominantly affecting the frontal lobes, the frontopolar, temporal cortex with some extension into the insula lobes, and other cortical regions (Fig. 1e-h).

A range of immunomodulatory plans have been used, in addition to the concomitant use of AEDs. Intravenous immunoglobulin at $0.4 \mathrm{~g} / \mathrm{kg}$ of for 5 days and/or intravenous methylprednisolone at $0.5-1 \mathrm{~g}$ per day for 5 consecutive days, with subsequent treatment, was used. Five of 6 patients showed improvement in seizure frequency after receiving immunotherapy but all relapsed after discontinuing methylprednisolone, and one continued to deteriorate and died. Post-QOLIE-31 shows that quality of life of who clinically respond to immunotherapy was improved in the last follow-up, and reduced antibody titers are observed in them but did not disappear.

\section{Discussion}

Acute symptomatic seizures related to GAD65 antibodies can be characterized by acute symptomatic seizures secondary to AE and autoimmune-associated epilepsy. Patients present with the former at the onset of the disease and then almost all develop into the latter. The difference is that the latter highlights that not only immune factors but also nonimmune factors (e.g., structural) are also present and may play a role in the development of seizures. Although our study was a small cohort, clinical manifestations range from only seizures to acute explosive encephalitis with new-onset refractory status epilepticus. This indicates the clinical heterogeneity in acute symptomatic seizures due to AE related to GAD65 antibodies.

Coexistence of anti-GAD65 and anti-LGI1 antibody is rare, and this results in the variability in the clinical and radiographic features. The tongue stiffness during the seizure indicates dystonia of the tongue muscle, and maybe it is a specific manifestation of faciobrachial dystonic seizure, which is a special feature of LGI1-AE [3]. Most patients with LGI1-AE can achieve seizure free without AEDs within 3-12 months after receiving immunotherapy [4]. Some scholars believe that antiGAD65 Abs may coexist with additional cell-surface antibody, and at this time, these antibodies might directly be pathogenic to cells, and anti-GAD65 antibodies might rather be a bystanding effect generated by bloodbrain barrier permeability. But, the dependence on the glucocorticoids suggests that anti-GAD65 antibodies may also play roles in the development and progression of the disease.

Different clinical characteristics were noted in different patients. We noted that diabetes in patient 1 and patient 2 showed a significant difference in prognosis even though they received the same treatment. Researchers [5] measured the antibody affinity of GAD65 antibody with DM and found that the responsiveness of GAD65 antibody was usually heterogeneous; this difference in antibody response may derive from different immune events, and this is what leads to different clinical characteristics in different patients.

Although there is a certain prevalence of positive antibodies and related diseases in the general population, the probability of positive antibodies and related diseases in AE patients is much higher than the background prevalence [6]. Previous studies [7] have shown that $1 \mathrm{AD}$ increases the chance of another $\mathrm{AD}$. In this study, in 2 patients, 2 ADs coexisted that tested this theory to some extent. Some scholars [8] speculate that vitiligo may be a clue to an autoimmune cause for AE; improvement of $\mathrm{AE}$ and vitiligo (Fig. 2a, b) after immunotherapy in patient 1 verifies the possibility.

Brain MRI of 2 patients in our cohort showed invasion of both the limbic lobes and cerebral cortex, which was inconsistent with the previous conclusion [9] that only the limbic lobes can be involved. Patient 2 who had no detection in repeated examinations explains that a negative brain MRI cannot rule out the disease.

A viral infection prodrome may precede the symptoms of AE. Niehusmann et al. [10] showed that the occurrence of GAD65 AE was related to human herpesvirus $6 \mathrm{~B}$ infection, which may be caused by viral infection causing the central nervous system epitope to be recognized by antibodies after exposure, resulting in the destruction of the body's autoimmune tolerance and the production of antiGAD65 antibodies. In our study, 3 patients had infection before seizure, which may trigger the occurrence of 


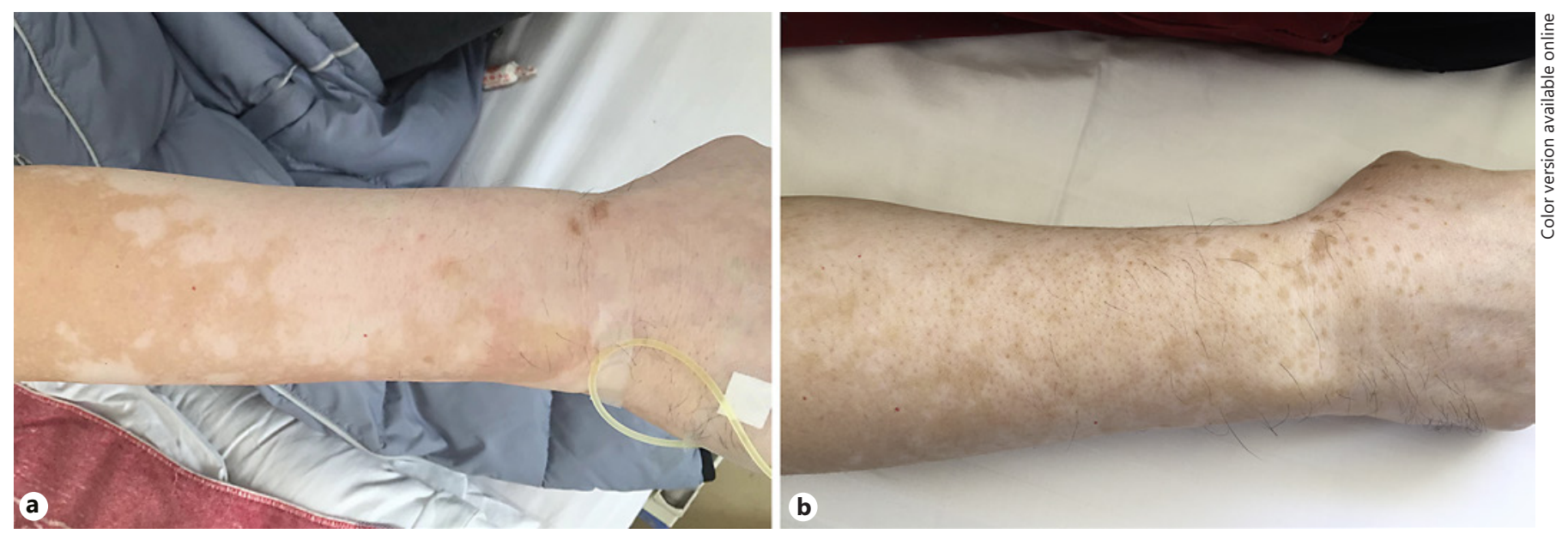

Fig. 2. a (Pt 4) The skin defect on the right forearm before immunotherapy. (Pt 4) The skin defect was improved 6 months after immunotherapy (b).

GAD65 AE. For patients with highly suspected autoimmune factors which caused the seizure, such as suspected viral encephalitis, adequate antiviral treatment is ineffective; immunotherapy can be applied experimentally before antibody positive detection.

The persistence of seizures after immunotherapy suggests an enduring predisposition, and this determines that long-term immunotherapy is required. This is consistent with what was mentioned earlier "autoimmune-associated epilepsy." There was no association between GAD65 antibody titer level and clinical severity of patients. We noted a decrease in antibody titers in patients who responded to immunotherapy but did not disappear in follow-up examination, which is similar to previous studies [11]. We suggest that the evaluation of clinical symptoms is more important in adjusting treatment.

In conclusion, our study reported clinical heterogeneity in acute symptomatic seizures due to AE related to GAD65 antibodies. It is necessary for patients to screen endocrinologic abnormalities and receive continuous immunotherapy.

\section{Statement of Ethics}

The study was approved by the Ethics Committee of China Japan Union Hospital of Jilin University. All patients were informed of the study and provided informed consent to participate in the study. Ethical Reference No. 20200008. Subjects (or their parents or guardians) have given their written informed consent.

\section{Conflict of Interest Statement}

None of the authors has any conflicts of interest to disclose.

\section{Funding Sources}

This study was supported by the Jilin Province Science and Technology Development Plan Project in China (Grant No. 20180311075yy).

\section{Author Contributions}

S. Liu contributed to conception and design; S. Wang and Y. Zhu contributed to collection and assembly of data; X. Fan and X. $\mathrm{Li}$ contributed to data analysis and interpretation; X. Fan contributed to writing of the manuscript; X. Han contributed to final approval of the manuscript.

\section{Data Availability Statement}

The data that support the findings of this study are openly available in PubMed at http://www.pubmed.net. All data generated or analyzed during this study are included in this article. Further enquiries can be directed to the corresponding author. 


\section{References}

1 Steriade C, Britton J, Dale RC, Gadoth A, Irani SR, Linnoila J, et al. Acute symptomatic seizures secondary to autoimmune encephalitis and autoimmune-associated epilepsy: conceptual definitions. Epilepsia. 2020;61(7): 1341-51.

2 Cramer JA. Quality of life for people with epilepsy. Neurol Clin. 1994 Feb;12(1):1-13.

3 Irani SR, Stagg CJ, Schott JM, Rosenthal CR, Schneider SA, Pettingill P, et al. Faciobrachial dystonic seizures: the influence of immunotherapy on seizure control and prevention of cognitive impairment in a broadening phenotype. Brain. 2013 Oct;136(Pt 10):3151-62.

4 Geis $\mathrm{C}$, Planagumà J, Carreño $\mathrm{M}$, Graus $\mathrm{F}$, Dalmau J. Autoimmune seizures and epilepsy. J Clin Invest. 2019;129(3):926-40.
5 Nakajima $\mathrm{H}$, Nakamura $Y$, Inaba $Y$, Tsutsumi C, Unoda K, Hosokawa T, et al. Neurologic disorders associated with anti-glutamic acid decarboxylase antibodies: a comparison of anti-GAD antibody titers and time-dependent changes between neurologic disease andtype I diabetes mellitus. J Neuroimmunol. 2018 Apr;317:84-9.

6 Zhao J, Wang C, Xu X, Zhang Y, Ren H, Ren $Z$, et al. Coexistence of autoimmune encephalitis and other systemic autoimmune diseases. Front Neurol. 2019;10:1142.

7 Zhernakova A, Withoff S, Wijmenga C. Clinical implications of shared genetics and pathogenesis in autoimmune diseases. Nat Rev Endocrinol. 2013;9(11):646-59. .
8 Haitao R, Huiqin L, Tao Q, Xunzhe Y, Xiaoqiu S, Wei L, et al. Autoimmune encephalitis associated with vitiligo? J Neuroimmunol. 2017;310:14-6.

9 Li X, Guo Q, Zheng Z, Wang X, Liu S. Immune-mediated epilepsy with GAD65 antibodies. J Neuroimmunol. 2020;341:577189.

10 Niehusmann P, Widman G, Eis-Hübinger AM, Greschus S, Robens BK, Grote A, et al. Non-paraneoplastic limbic encephalitis and central nervous HHV-6B reactivation: causality or coincidence? Neuropathology. 2016; 36(4):376-80.

11 Kwan P, Sills GJ, Kelly K, Butler E, Brodie MJ. Glutamic acid decarboxylase autoantibodies in controlled and uncontrolled epilepsy: a pilot study. Epilepsy Res. 2000;42(2-3):191-5. 